\title{
Caso raro de hemoptisis por hemangioma lobular capilar traqueal
}

\author{
Eduardo Leyva-Moraga,* $\bowtie$ Jesús Martín Ibarra-Celaya, ${ }^{\ddagger}$ \\ Graciano Castillo-Ortega, ${ }^{\S}$ Francisco Alberto Leyva Moraga*
}

*Universidad de Sonora, Hermosillo, México; ${ }^{\ddagger}$ Hospital General de Zona No. 2, Hermosillo, México;

${ }^{\S}$ Hospital General del Estado de Sonora, Hermosillo, México.

\begin{abstract}
RESUMEN. Los hemangiomas capilares son tumores benignos que se encuentran normalmente en mucosas y piel. Con rareza estos tumores pueden llegar a ser localizados en tráquea y en la vía aérea central. Presentamos un caso de resección y anastomosis de tráquea intratorácica por hemoptisis recurrente secundario a hemangioma lobular capilar. Paciente masculino de 47 años que fue referido a nuestro centro por hemoptisis recurrente secundaria a tumor endofítico en tráquea distal a $3.5 \mathrm{~cm}$ de la carina. Decidimos que el paciente requería un abordaje por toracotomía posterolateral para la resección traqueal basándonos en la localización anatómica del tumor. El reporte histopatológico definitivo reveló un diagnóstico de hemangioma capilar lobular.
\end{abstract}

Palabras clave: Hemangioma lobular capilar, tráquea distal, tumor traqueal.

\section{Rare case of hemoptysis due to tracheal lobular capillary hemangioma}

ABSTRACT. Capillary hemangiomas are benign tumors that are commonly found in mucous membranes and cutaneous surfaces. Rarely, these tumors can be located in the trachea and central airway. We present a case report of intrathoracic tracheal resection and anastomosis for recurrent hemoptysis due to capillary hemangioma. A 47-year-old male patient was referred to our center for recurrent hemoptysis secondary to a distal endophytic tracheal tumor with a distance of $3.5 \mathrm{~cm}$ to the carina. We used a posterolateral approach to perform the tracheal resection based on the tumor anatomical position. The histopathological report revealed lobular capillary hemangioma.

Key words: Distal trachea, lobular capillary hemangioma, tracheal tumor.

\section{INTRODUCCIÓN}

Los tumores primarios de tráquea son raros, representan 0.1-0.4\% de los tumores de la vía respiratoria; usualmente son malignos en adultos y benignos en niños. ${ }^{1}$ Por lo general, se presentan con signos de obstrucción central y su diagnóstico es retardado, ya que tienden a ser tratados como asma. ${ }^{2-4} \mathrm{El}$ hemangioma capilar lobular $(\mathrm{HCL})$ es un tumor benigno que se presenta comúnmente en piel y mucosas de la vía aérea superior caracterizado por un crecimiento rápido y una superficie friable. ${ }^{5}$

Hasta el momento no se conoce adecuadamente el comportamiento del HCL en esta localización por la

\section{$\triangle$ Autor para correspondencia:}

Dr. Eduardo Leyva-Moraga, Universidad de Sonora, Hermosillo, México.

Correo electrónico: leyvamoragaeduardo@gmail.com

Trabajo recibido: 22-X-2018; aceptado: 18-I-2019 poca información disponible. Presentamos el caso de un paciente masculino de 47 años con HLP localizado en tráquea, tratado con resección quirúrgica por toracotomía lateral.

\section{REPORTE DE CASO}

Masculino de 47 años, fumador, referido a nuestro centro hospitalario por la presencia de tumor intratraqueal con hemoptisis de 3 días seguidos de $500 \mathrm{ml} / \mathrm{día}$, misma que cedió espontáneamente y de 15-20 eventos subsecuentes de $100 \mathrm{ml} /$ día en los últimos seis meses. Estos eventos fueron manejados en otro centro hospitalario en donde se realizó tomografía torácica (figura 1) y biopsia por broncoscopia flexible, con resultado patológico de tejido inflamatorio necrótico.

En nuestro centro se repitió la broncoscopia (figura 2) encontrando tráquea de aspecto empedrada con poca visibilidad de anillos traqueales y un tumor acartonado, friable y sangrante, localizado en cara anterior de trá-

Este artículo puede ser consultado en versión completa en: www.medigraphic.com/neumologia 


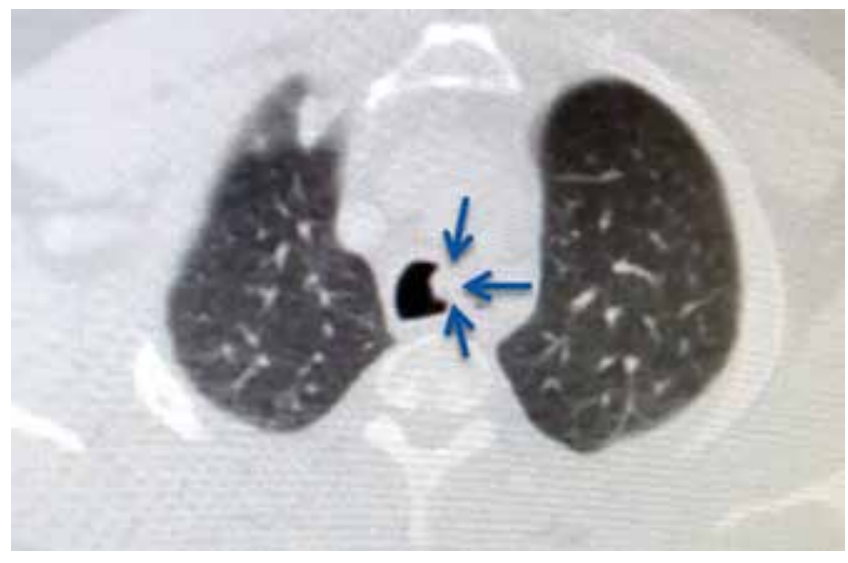

Figura 1: Tomografía computarizada de corte axial de pulmón, en donde se evidencia (flecha) tumor intratraqueal.

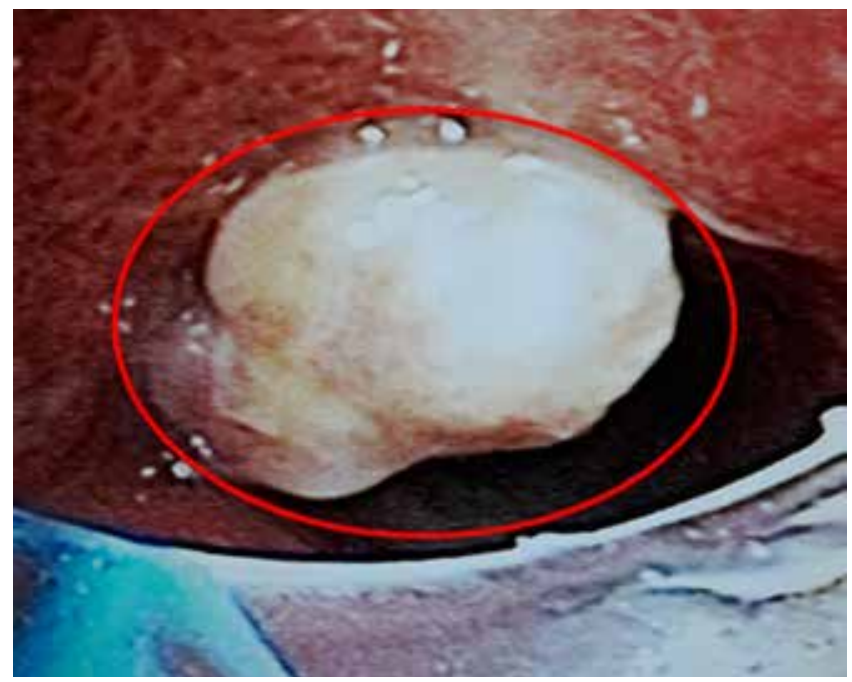

Figura 2: Broncoscopia al ingreso del paciente. Tráquea empedrada y tumor endofítico (circulado).

quea a $3.5 \mathrm{~cm}$ de la carina de aproximadamente $0.5 \mathrm{x}$ $1.0 \mathrm{~cm}$. Se realizó resección de $60 \%$ de la tumoración y hemostasia con adrenalina tópica. El resultado fue idéntico al de la primera biopsia. El paciente continuó con episodios de hemoptisis de 50-80 ml/día, por lo que se realizó una nueva broncoscopia cinco días después, observando tumoración de las mismas características $(0.5 \times 1.0 \mathrm{~cm})$ sangrante. Se decide resección quirúrgica de segmento traqueal afectado.

\section{ABORDAJE QUIRÚRGICO}

Se realizó resección de tráquea por toracotomía posterolateral derecha. El paciente requirió intubación selectiva del bronquio principal izquierdo para colapsar pulmón derecho. El abordaje fue por el cuarto espacio intercostal donde se disecó y ligó la vena ácigos para proceder a la disección de tráquea, por medio de broncoscopio se delimitó el área de resección tumoral (figura 3). La disección abarcó los 3-4 anillos contenedores del tumor (figura 4), dejando márgenes de $1 \mathrm{~cm}$ superior e inferior. Se procedió a anastomosis terminoterminal realizada con vycril 3-0 (poligactina 910) puntos simples separados y reforzada con pleura vascularizada y cianoacrilato. Para verificar la ausencia de fuga se realizó maniobra de Valsalva a $40 \mathrm{~cm}$ de $\mathrm{H} 20$.

Una semana después se realiza broncoscopia de control que mostró una anastomosis íntegra y sin fugas. El resultado histopatológico de la pieza quirúrgica reveló un diagnóstico de hemangioma lobular capilar. El paciente fue dado de alta sin complicaciones, sin datos de estridor ni dificultad respiratoria. En la actualidad está en el tercer mes de seguimiento y clínicamente asintomático.

\section{DISCUSIÓN}

El HLC localizado en tráquea es una entidad que se ha descrito pocas veces en la literatura. Ésta es la primera vez que se describe en México.

Los dos síntomas más comunes del HLC traqueal son tos y hemoptisis, la cual puede variar de menor a mayor grado. ${ }^{5}$ Nuestro paciente sufrió de eventos esporádicos

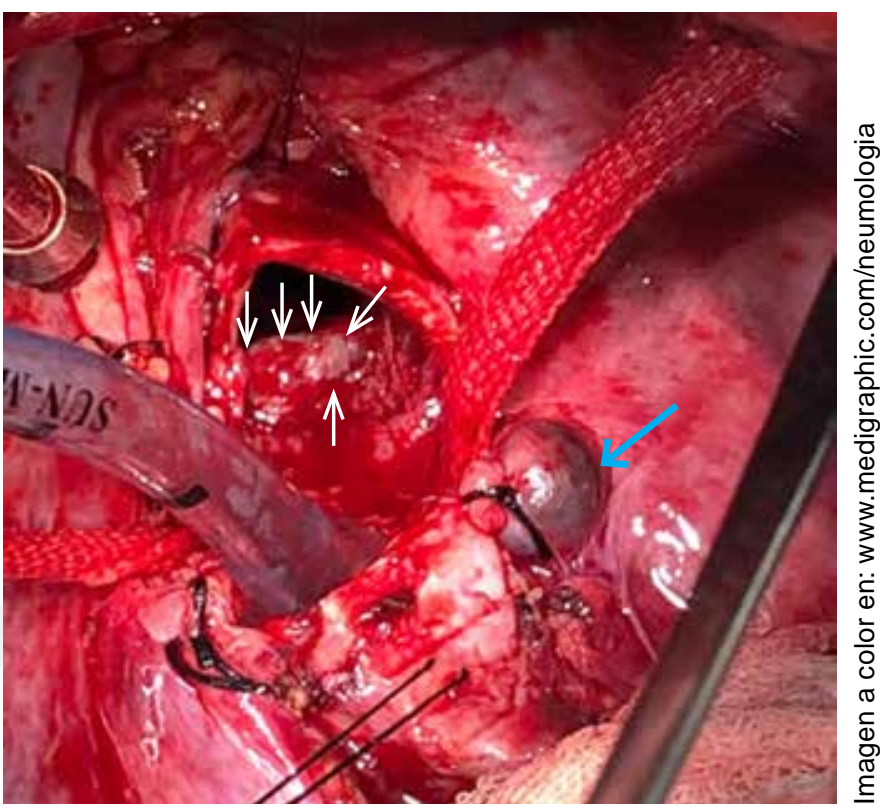

Figura 3: Resección de tráquea. Se puede observar tumoración en el segmento superior (flechas blancas) y vena ácigos ligada lateral a la tráquea (flecha azul). 


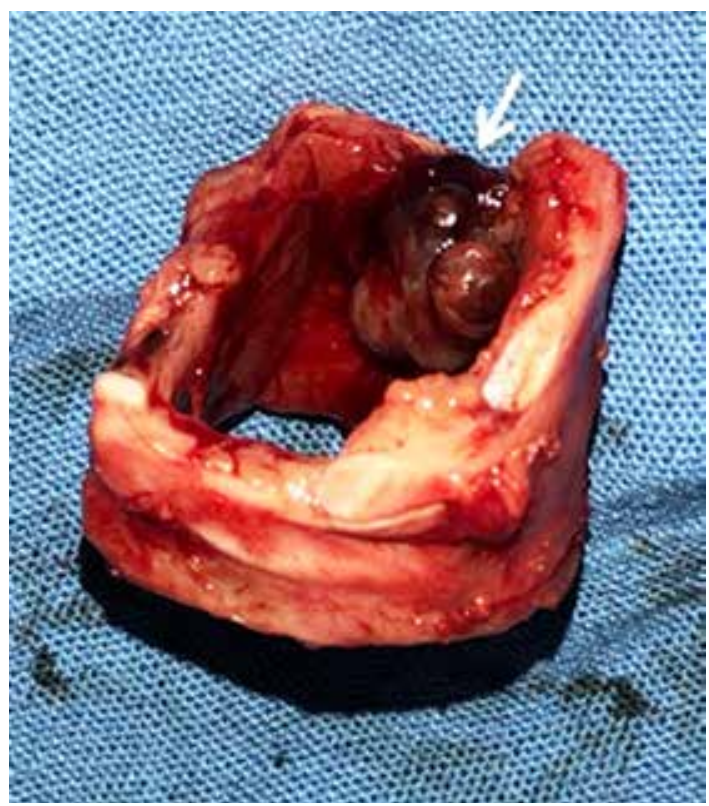

Figura 4: Anillos traqueales resecados y tumor traqueal color marrón (flecha).

de hemoptisis mayor que pusieron en riesgo su vida.

La etiología del HLC no se ha definido por completo. Sin embargo, existen postulados que asocian esta patología con cambios hormonales, trauma, infecciones virales o bacterianas, producción de factores angiogénicos y deleciones citogenéticas clonales. ${ }^{6}$

Al día de hoy no se ha llegado a un consenso del mejor tratamiento del HLC traqueal, pero se han descrito algunas opciones terapéuticas; entre ellas, la broncoscopia flexible o rígida con diferentes modalidades como crioablación, electrocauterización, fotocauterización, ${ }^{5-9}$ teniendo excelentes resultados con poca morbilidad. Otra alternativa descrita en un reporte de un caso chino realizado en el 2012 fue la braquiterapia, que pudo ofrecer grandes ventajas al ser un tratamiento localizado. ${ }^{10}$

En nuestro caso, el paciente requirió resección quirúrgica aun sin un resultado concluyente de histopatología por la recurrencia y magnitud del sangrado de vía aérea, así como por el pobre control logrado por broncoscopia. El abordaje transtorácico para resección y anastomosis de tráquea es raro (2.04\%). ${ }^{11}$ En el caso que presentamos debido a que la localización de la tumoración era complicada para un abordaje cervical, se tomó la decisión de realizar toracotomía lateral para proceder con la resección, teniendo un excelente resultado en la disección del segmento traqueal constituido por el tumor.

\section{Conflicto de intereses}

Los autores declaran no tener conflicto de intereses.

\section{REFERENCIAS}

1. Grillo HC, Mathisen DJ. Primary tracheal tumors: treatment and results. Ann Thorac Surg 1990;49(1):69-77.

2. Macchiarini P. Primary tracheal tumours. Lancet Oncol 2006;7(1):83-91.

3. Ayadi-Kaddour A, Khadhar A, Mlika M, et al. Une tumeur trachéale traitée comme un asthme. Rev Pneumol Clin 2014;70(6):353-356. doi: 10.1016/j. pneumo.2014.04.003.

4. Schneider P, Schirren J, Muley T, Vogt-Moykopf I. Primary tracheal tumors: experience with 14 resected patients. Eur J Cardiothoracic Surg 2001;20(1):12-18.

5. Dabó H, Gomes R, Teixeira N, Teixeira G, Fernandes G, Magalhães A. Tracheal lobular capillary hemangioma treated with laser photocoagulation. J Bras Pneumol 2016;42(1):72-73. doi: 10.1590/S180637562016000000217.

6. Chen E, Yu X, Zhang R, Wang P, Ying K. A large tracheal capillary hemangioma treated with interventional bronchoscopy. Respiratory Medicine CME 2011;4(2):6061. doi: 10.1016/j.rmedc.2010.10.0 02

7. Amy FT, Enrique DG. Lobular capillary hemangioma in the posterior trachea: a rare cause of hemoptysis. Case Rep Pulmonol 2012;2012:592524. doi: 10.1155/2012/592524.

8. Porfyridis I, Zisis C, Glinos K, et al. Recurrent cough and hemoptysis associated with tracheal capillary hemangioma in an adolescent boy: a case report. J Thoracic Cardiovasc Surg 2007;134(5):1366-1367.

9. Chawla M, Stone C, Simoff MJ. Lobular capillary hemangioma of the trachea: the second case. J Bronchology Interv Pulmonol 2010;17(3):238-240. doi: 10.1097/LBR.0b013e3181e65117.

10. Jie S, Hong-Rui L, Fu-Quan Z. Brachytherapy for tracheal lobular capillary haemangioma (LCH). J Thorac Oncol 2012;7(5):939-940. doi: 10.1097/JTO.0b013e31824cc493.

11. Hecker E, Volmerig J. Extended tracheal resections. Thorac Surg Clin 2014;24(1):85-95. doi: 10.1016/j. thorsurg.2013.10.005. 